\title{
Companion of choice at birth: factors affecting implementation
}

Tamar Kabakian-Khasholian ${ }^{1 *}$ and Anayda Portela ${ }^{2}$

\begin{abstract}
Background: Two recent recommendations made by the World Health Organization confirm the benefits of companion of choice at birth on labour outcomes; however institutional practices and policies do not always support its implementation in different settings around the world. We conducted a review to determine factors that affect implementation of this intervention considering the perspectives and experiences of different stakeholders and other institutional, systemic barriers and facilitators.

Methods: Forty one published studies were included in this review. Thirty one publications were identified from a 2013 Cochrane review on the effectiveness of companion of choice at birth. We also reviewed 10 qualitative studies conducted alongside the trials or other interventions on labour and birth companionship identified through electronic searches. The SURE (Supporting the Use of Research Evidence) framework was used to guide the thematic analysis of implementation factors.

Results: Women and their families expressed appreciation for the continuous presence of a person to provide support during childbirth. Health care providers were concerned about the role of the companion and possible interference with activities in the labour ward. Allocation of resources, organization of care, facility-related constraints and cultural inclinations were identified as implementation barriers.

Conclusion: Prior to introducing companion of choice at birth, understanding providers' attitudes and sensitizing them to the evidence is necessary. The commitment of the management of health care facilities is also required to change policies, including allocation of appropriate physical space that respects women's privacy. Implementation research to develop models for different contexts which could be scaled up would be useful, including documentation of factors that affected implementation and how they were addressed. Future research should also focus on documenting the costs related to implementation, and on measuring the impact of companion of choice at birth on care-seeking behavior for subsequent births.
\end{abstract}

Keywords: Companion of choice at birth, Continuous support during childbirth, Labour companionship, Lay companion, Doula, Implementation

\section{Background}

Companion of choice at birth is defined as the continuous presence of a support person during labour and birth [1]. The intervention has been recommended by the World Health Organization (WHO) to improve labour outcomes and women's satisfaction with care $[2,3]$. It has also been identified as a key element in the WHO vision of quality of care for pregnant women and newborns [4]. Different names have been given to the intervention including

\footnotetext{
* Correspondence: tk00@aub.edu.lb

${ }^{1}$ Health Promotion and Community Health Department, Faculty of Health

Sciences, American University of Beirut, Beirut, Lebanon

Full list of author information is available at the end of the article
}

continuous support during childbirth, companion of choice at birth, labour companion, emotional support during birth. We will refer to this intervention herein as companion of choice at birth.

Evidence for companion of choice at birth emanates from a Cochrane systematic review conducted in 2013 [1] showing that companion of choice at birth increases the likelihood of vaginal births, therefore reduces the need for caesarean sections and the use of forceps or vacuum during vaginal births. In addition, it reduces the need to use pain medications during labour, it shortens the duration 
of labour and improves women's satisfaction with care. It also improves Apgar scores of the newborns.

The Cochrane systematic review included 22 trials involving more than 15,000 women. The trials were conducted in low, middle and high-income countries with considerable variation in hospital settings. The form of care evaluated was continuous presence and support during labour or labour and birth. The control group received "usual care" as defined by the study investigators that did not involve continuous support during labour or during labour and birth.

In the research conducted, the support person varied from a nurse or midwife of the hospital staff to a doula, a woman not related to the labouring woman and not a hospital staff but employed through the trial to accompany labouring women, to a companion of choice identified by the pregnant woman from her social network. The most beneficial form of support appears to be from a person who is not a member of the woman's social network, is not hospital staff and who has some experience or has received some informal training. However, in the absence of such a person, the support from a person of choice from among the woman's family or friends improves women's satisfaction with care [1].

Institutional routines and policies, providers' attitudes as well as the physical environments of hospitals do not always support the implementation of the intervention. Given the multiple dimensions of this intervention including variation in the delivery of the intervention according to the context and the provider of the support, there is a need for greater understanding of the factors that influence successful implementation and sustainability of the intervention. These factors include the perspectives, experiences, knowledge and skills of different stakeholders involved in implementation, as well as relevant decision making processes, and barriers and facilitators within and outside of the health facility.

This paper aims at a) describing stakeholder perspectives and experiences with the intervention; b) identifying the barriers and facilitating factors to implementation; and c) describing those implementation factors which could be linked to programmes who reported on increased women's satisfaction.

\section{Methods}

This is a literature review of factors influencing implementation of companions of choice at birth. It is based on information describing the implementation of the intervention derived from trials identified through the Cochrane review [1], qualitative research conducted alongside any of these trials, or studies describing health care providers' attitudes towards the provision of support during childbirth.
This review included 41 publications. We assessed all the studies identified in the Cochrane review in 2013 [1] for reported information on implementation factors. These also included studies that were excluded from the Cochrane review [1]. We included 31 studies in our review through this process:22 published articles [5-26] included in the meta-analysis of the Cochrane Review [1] and 9 excluded from it [27-35]. We excluded one of the trials included in the Cochrane review [1] because it was not published in English [36]. In addition, we conducted electronic searches on Pubmed and Medline to identify qualitative studies describing the experiences of stakeholders with the implementation of companion of choice at birth published through March 2015. We also searched the bibliographies of the published trials to identify qualitative studies on implementation factors or feasibility of the intervention published alongside the trials. We identified an additional 10 studies in this category [37-46].

No quality assessment of studies was done for this review, considering that we were interested in identifying publications reporting on implementation factors that were not directly related to validity of the outcomes assessed in the trials.

The first author extracted data from the publications included in this review, and used the SURE (Supporting the Use of Research Evidence) framework [47] to guide the extraction and categorization of information on implementation factors into meaningful themes. A research assistant verified the coding and abstracting of the data. Thematic analysis was used to identify factors that influence implementation of the intervention as well as different stakeholder perspectives and experiences of the intervention and contextual characteristics. To capture information about the context of the implementation in the reviewed studies, we retrieved information from the discussion sections. The variation in settings in these studies between high, middle and low income countries allowed for some comparison of factors in the different contexts.

\section{Results}

The characteristics of the studies included in this analysis are presented in Table 1. Among all the reviewed studies, only eight aimed at reporting on implementation factors for the introduction of a companion of choice at birth in a hospital $[13,23,27,32,43-46]$. The remaining studies in this review aimed at reporting the effectiveness of the practice.

All identified implementation factors are presented in Table 2.

\section{Stakeholders' perspectives}

Studies mainly reported on the experiences and perspectives of women and health care providers. Receiving 






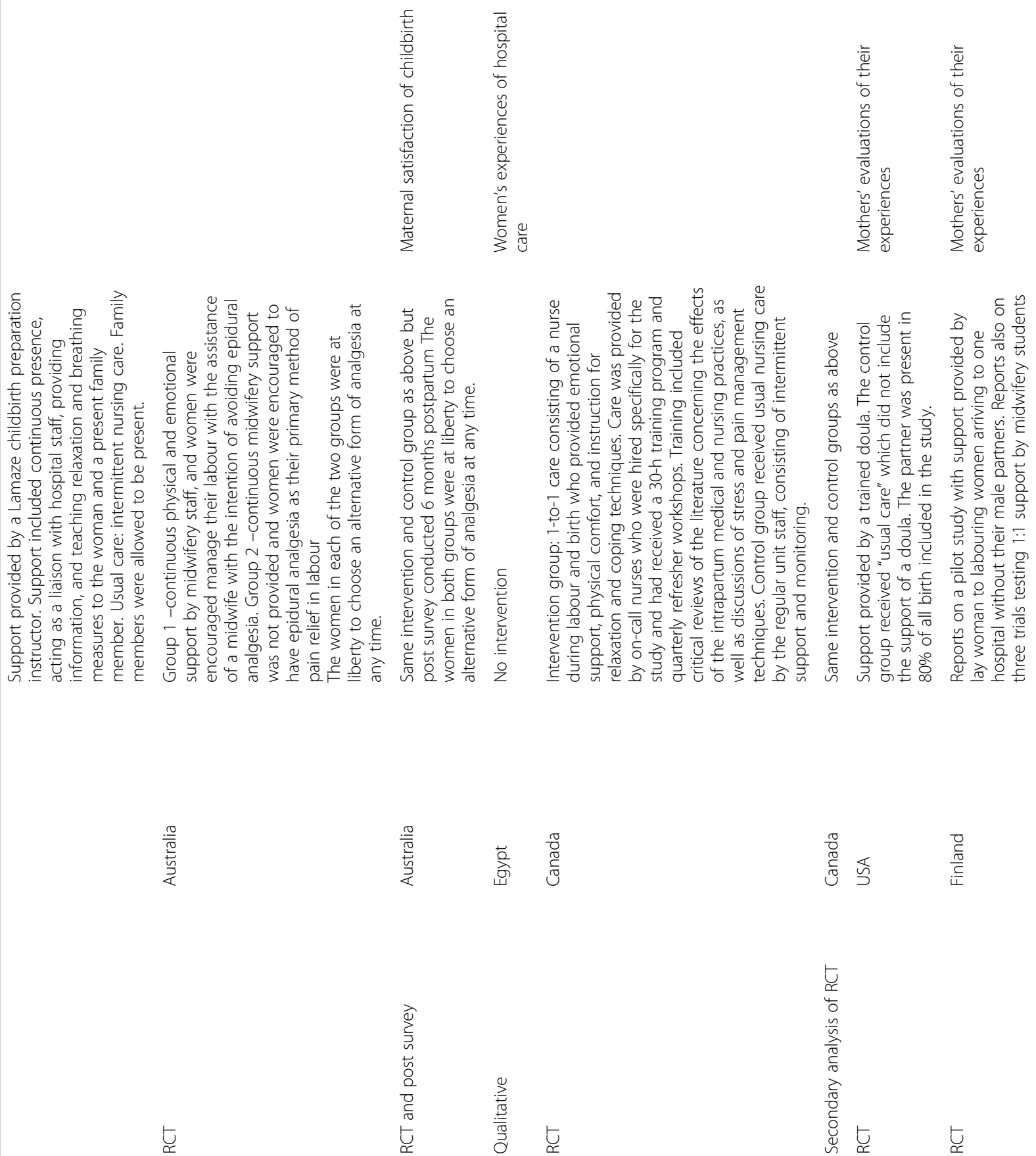

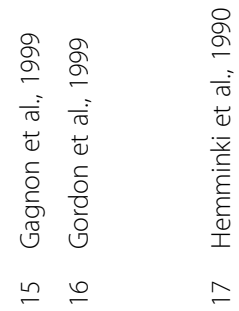



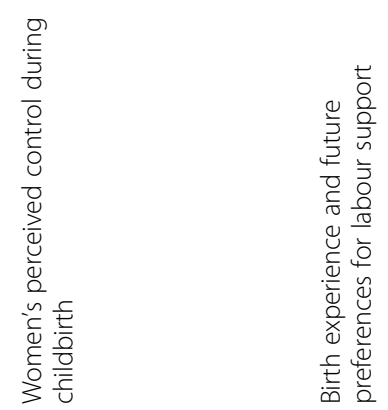

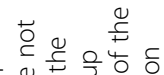

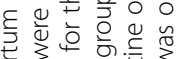

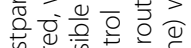

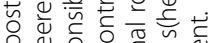

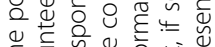

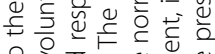

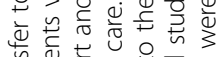

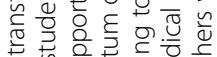

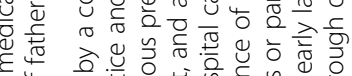

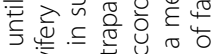

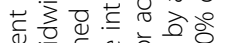
हैं है.

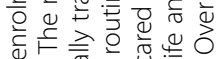

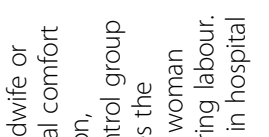

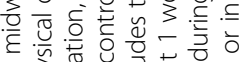
त्री

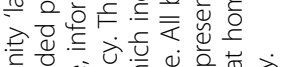

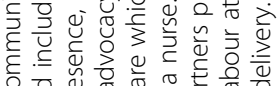
हो.

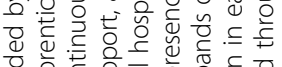

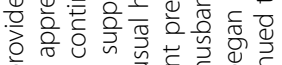

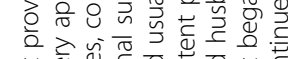

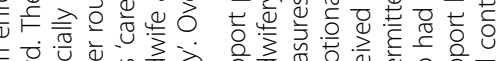

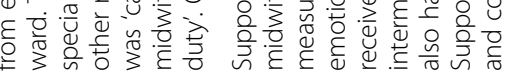

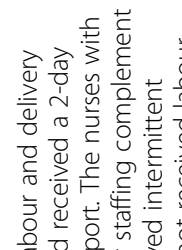

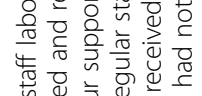

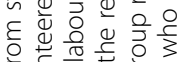

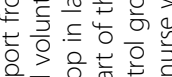

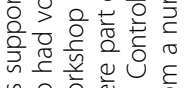

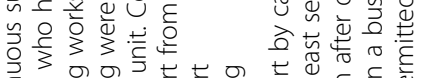

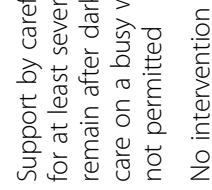

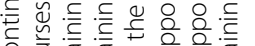

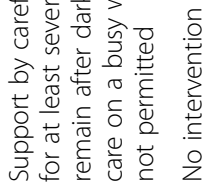

票

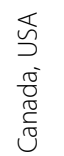



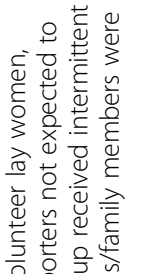



둔

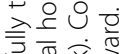

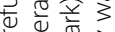
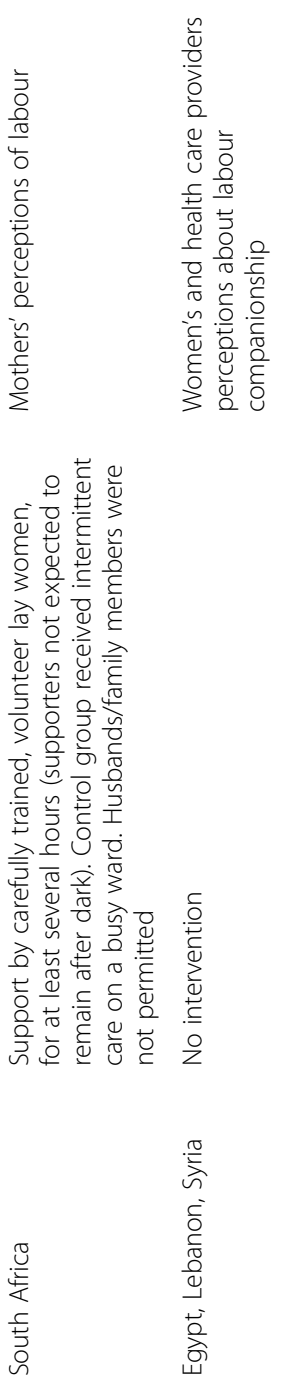

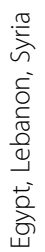

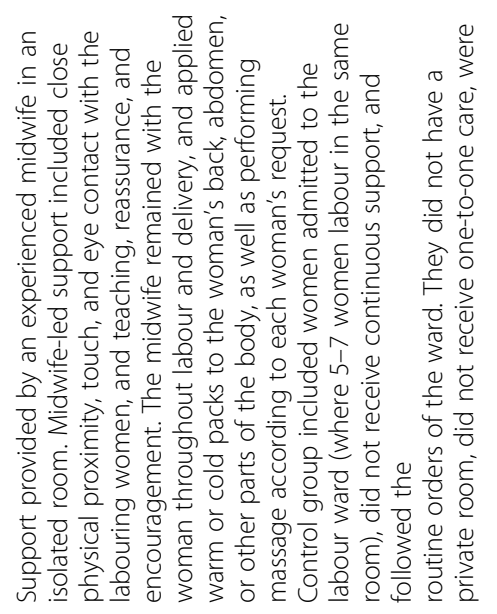

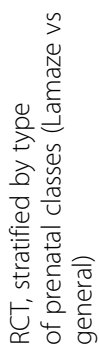

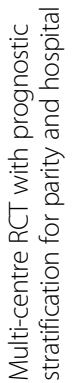

뜬

$\underset{\Upsilon}{\longleftarrow}$

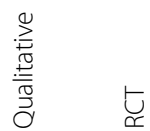

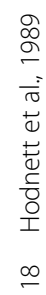

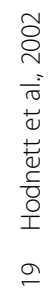

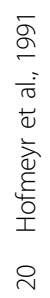

高

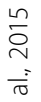

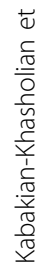

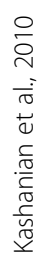

$\bar{\sim} \approx$ 






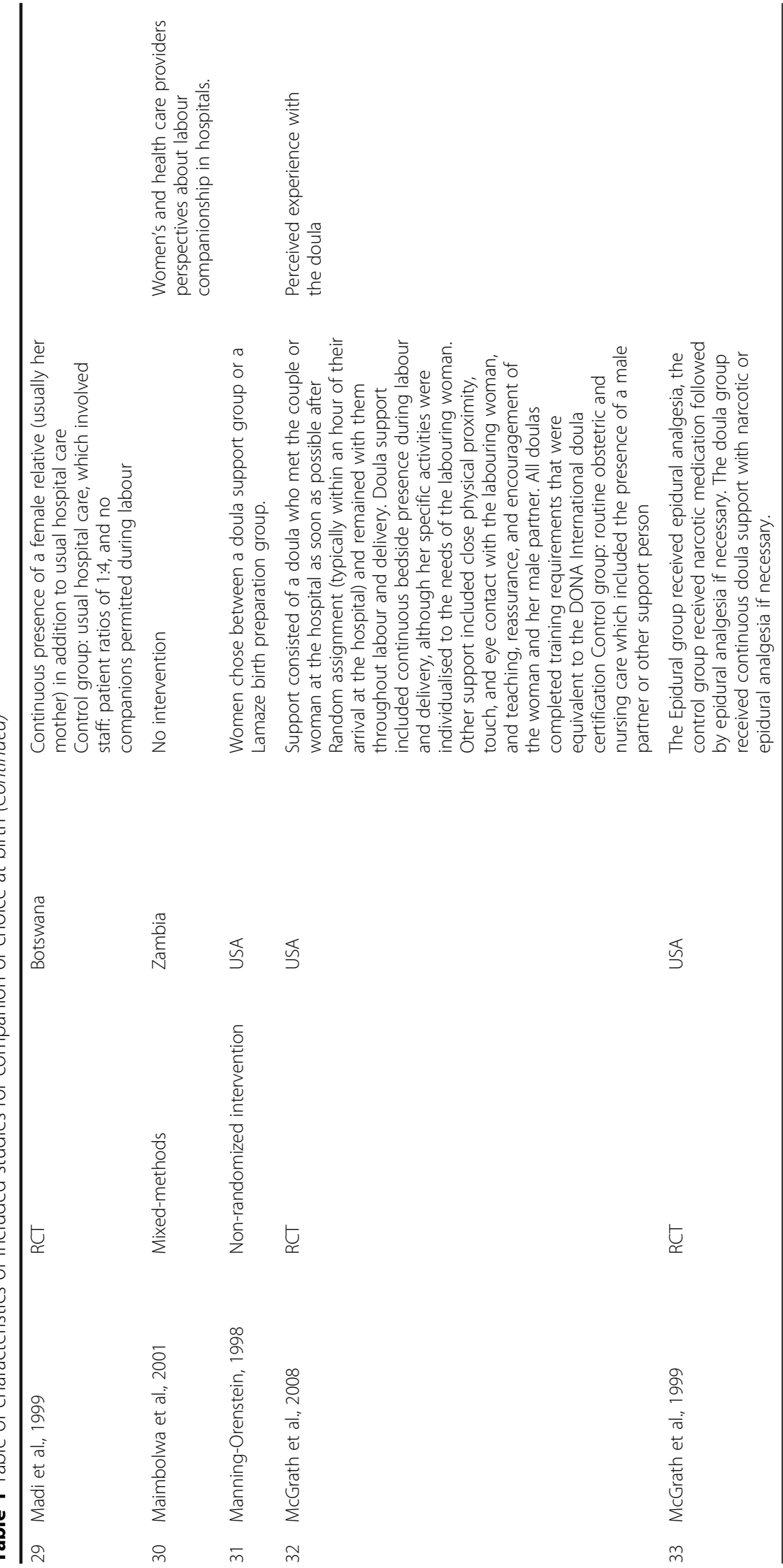







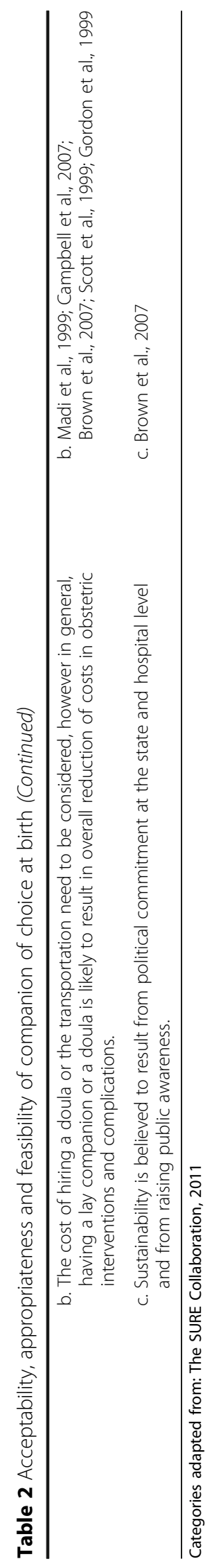


support by a companion of choice at birth was reported as a positive experience that was largely appreciated by women, and when present, by their male partners [13$15]$. In three other studies, the presence of the companion was experienced as a buffer to women's and their families' low expectations and experiences of bad treatment at hospitals [16, 27, 43].

In some close-knit communities, where most people knew each other, women were reserved about the presence of doulas recruited from their same community or the presence of a female family member with them during childbirth [39, 42, 45]. They expressed their worries of being exposed to the companion and the consequent expected gossip in the community about them not being able to keep up with social expectations of behaviour, such as not losing control and not shouting. This is in contrast to another low-income community where lay female companions were regarded as allies not invested in the hospital system and their presence was appreciated [17].

In three studies conducted in high-income countries and one from a low-income setting, the presence of a female companion was perceived to be beneficial in addition to the presence of male partners. Despite the fact that male partners were appreciated for providing emotional and spiritual reassurance, women perceived them as lacking the skills needed for other aspects of support necessary to them $[14,18,32,45]$ as well as not coping well with seeing women go through labour and birth [45]. Both in low and high income country settings, male partners were found to be not consistently present throughout labour, they were mainly absent in early and late labour and were viewed as being less physically interactive compared to female companions [32, 45]. In conservative cultures, such as in Arab countries, in Ghana, and in China, women sometimes appreciated the presence of the male partner to witness the challenges that women go through during childbirth [43-45]. In Arab cultures, the presence of the male partners was not socially acceptable especially in shared labour rooms [40, 43].

The attitudes of health care providers involved in the intervention were sometimes positive as they perceived the presence of the companion helpful in reducing the dependency of women on the staff. This was mainly important in settings with shortage of nursing and midwifery staff $[16,21,39]$. The presence of the companion was noted to positively influence the behaviour of the staff towards women [21]. Some studies reported negative attitudes and some resistance in acceptance of companions to labour wards $[13,20,39,44,46]$. Nurses and midwives reported their doubts about the role of the companion and expected less cooperation of women with the staff throughout labour and birth in the presence of the companion [13, 20,39,44,46]. Concerns were reported about the use of traditional medicines by lay companions [39]. Providers also reported concerns about companions who are not part of the hospital staff, interfering in medical decisions and about cross infections in the labour and delivery ward [39, 43, 44]. Efforts such as influencing the attitudes of health care providers by informing them about the evidence on companion of choice at birth and motivating them by sharing positive birth experiences are considered to be necessary for the successful implementation of this practice [21, 22].

\section{Barriers and facilitating factors for implementation}

A number of health service-related barriers and facilitating factors were identified in the implementation of companion of choice at birth in hospital settings. These varied according to resources available in the facility and to the people providing support. Whereas having a female relative as a companion is considered a low cost intervention that is valued in facilities with shortage of nursing staff [16, 25, 39], the task of providing continuous support by nurses and midwives of the hospital was reported to intensify the human resource shortages [39] or had implications on the organization of shifts at the labour ward [27]. Where doulas were considered as labour or birth companions, their arrival to the labour ward early during labour was deemed necessary, for example before the woman had taken the decision to ask for an epidural [28].

The presence of an accompanying person was also found to have implications for the organization of space in the facility. In low or middle income country hospitals, a lounge near the labour ward was deemed necessary for companions to take short breaks [25]; in others providing a private space or a cubicle for the dyad of the labouring women and her companion was considered to be important for the success of the intervention [27, 39, $43,44]$. Crowding of shared labour rooms was a major concern in resource-constrained facilities [16, 43].

In facilities with shortage of nurses or midwives, the possibility of hiring and training unemployed or retired nurses and midwives was suggested [24, 26, 45] as well as training of lay companions [22, 43, 45] or doulas [23]. Clear communication with the companion about his/her duties was deemed necessary to reduce the negative providers' perceptions about the role of the companion $[43,46]$.

Although none of the reviewed studies reported cost analysis, there were some discussion on expected cost implications in few of these studies. In high-income countries and among high-income populations, it is expected that the cost of hiring a doula will be covered by women themselves as very few hospital-based programmes offer that option [22]. In low-income settings, the involvement of family members as companions during childbirth is an inexpensive practice [16] however, 
the cost of transportation for volunteer companions need to be considered to sustain the programme at the facility [27]. In general, it is expected that the beneficial effect of a companion of choice at birth would be costeffective for healthcare insurers and for hospitals as it reduces the financial costs of obstetric interventions such as epidural use and cesarean sections and the time spent on complications [16, 32].

\section{Discussion}

Women reported positive experiences with having a companion of choice at birth across the different facility settings and country-income levels in the reviewed studies, regardless of the person providing the support. In facilities where male partners' presence was accepted in addition to the female companion, an added value of the female companion was reported. Whereas the evidence indicates that companion of choice at birth is mostly an effective intervention when provided by persons who are not employed by the hospital [2], considerations should be given to the acceptance of doulas or family members in each cultural context as well as to the entailed costs for training or transportation.

We note the importance of the person providing the support in terms of increased women's satisfaction with the birth experience. Only four studies reported on this outcome [7, 21, 23, 24]. Two studies that included lay female relatives of labouring women as companions reported an increased satisfaction of women during childbirth [21, 23]. The effectiveness of the intervention was attributed by the authors to the fact that the companions were related to women therefore more in tune with women's needs [23] and to the positive influence of their presence on health care providers' behaviours in terms of sharing of information related to the care given to the labouring woman [21]. Another study showing an increase in women's satisfaction also reported the provision of information as a positive factor influencing women's satisfaction, however in this case the support during childbirth was provided by midwives [7]. One trial in Mexico that used retired nurses as companions during childbirth reported no effect on women's satisfaction [24]. Retired nurses were considered to be "de-sensitized" to women's needs and less empathetic than lay companions [24].

Providers' perceived the usefulness of the companions in facilities suffering from nurse and midwife shortage. In all of studies conducted in high or low income countries, providers carried negative attitudes towards the implementation of companion of choice at birth. They reported concerns about cross infection and crowding in labour wards as well as about the expected collaboration of women and of companions with the healthcare team and feared interferences with clinical decisions.
Other implementation barriers existed such as the allocation of resources within the facility and the organization of care to facilitate nurses or midwives continuous presence with the woman during labour and birth. Barriers in resource constrained environments related mainly to crowding and availability of space and privacy for women and their companions in the labour ward as well as to cultural preferences of the companion.

The implementation of this practice requires the commitment of the management of health care facilities to change institutional policies and to provide the appropriate physical space that respects women's and their companion's privacy. This could be informed by recent literature on the importance of supporting the supporters during childbirth through observing their interaction with the space [48]. While the evidence doesn't indicate the necessity of providing training to the lay companion [2], there were different approaches used in the reviewed interventions and considerations need to be given to various modes of orienting the companion in order to improve the acceptance of the intervention mainly by the health care providers.

Influencing the attitudes of health care providers is necessary for the successful implementation of the intervention. This could be achieved through sensitization activities including the provision of evidence-based information, through minimizing system barriers such as to avoid overloading the staff and resolving issues of space and privacy, through affecting providers' behaviours and through sharing of women's positive experiences with this practice to motivate their participation. The latter can be achieved through the use of effective communication to clarify the role of the companion in the labour and delivery wards.

Given the improved health outcomes reported as well as the positive experiences of women with companion of choice at birth reported regardless of the person providing support across all studies, we had expected that the use of this practice in hospitals would increase facility births. However, no trials reported on the effect of this practice on this outcome. Two studies reported on women's views reported that being denied of having a companion during childbirth can act as a reason for women opting to give birth at home [38, 39]. The need for such specific studies in those contexts where facility births are low would confirm if increased quality and increased satisfaction with services increases use of the services.

Implementation factors were mostly described in the qualitative studies identified for this review. These studies were descriptive in nature and focused on women's experiences and views [37, 38, 41, 45], few also included the perspectives of health care providers and discussed system related factors important for implementation $[39,43,46]$. 


\section{Conclusion}

More effort should be put in documenting and reporting implementation factors associated with interventions, by describing the context in terms of facility practices, providers' attitudes, community culture and challenges, either within the reporting of the intervention outcomes or in separate publications. More importantly, future studies should use implementation research methods to develop and test companionship models in different facility settings and cultures. This will inform initiatives that aim at for scaling-up these models of care to a larger number of health care settings in their region or country.

Future research should also focus on measuring the costs pertaining to the training of personnel or the lay companion and to the changes in the physical space, as well as on the impact of companion of choice at birth on care seeking behavior for subsequent births.

\section{Acknowledgements}

The authors thank Hannah Bontongton and Sylvana Hassanieh for their assistance in preparing this manuscript and Helen Smith for her guidance and valuable comments.

\section{Funding}

Funded by the World Health Organization through the work done for the development of $\mathrm{WHO}$ recommendations on health promotion intervention for maternal and newborn health 2015.

\section{Availability of data and materials}

Data sharing is not applicable to this article as no datasets were generated or analysed during the current study.

\section{Authors' contributions}

TKK carried out the analysis and drafted the manuscript. AP contributed in the interpretation and to the writing of the manuscript. AP is a staff member of the World Health Organization. She alone is responsible for the views expressed in this article and they do not necessarily represent the decisions, policy or views of the World Health Organization. Both authors read and approved the final manuscript.

\section{Consent for publication}

Not applicable

\section{Ethics approval and consent to participate}

Not applicable

\section{Competing interests}

The authors declare that they have no competing interests.

\section{Publisher's Note}

Springer Nature remains neutral with regard to jurisdictional claims in published maps and institutional affiliations.

\section{Author details}

${ }^{1}$ Health Promotion and Community Health Department, Faculty of Health Sciences, American University of Beirut, Beirut, Lebanon. ${ }^{2}$ Department of Maternal, Newborn, Child and Adolescent Health, World Health Organization, Geneva, Switzerland.
Received: 21 December 2015 Accepted: 4 August 2017

Published online: 31 August 2017

\section{References}

1. Hodnett ED, Gates S, Hofmeyer GJ, Sakala C. Continuous support for women during childbirth. Cochrane Database Syst Rev. 2013;7:CD003766. doi:10.1002/14651858.CD003766.pub5.

2. WHO recommendations for augmentation of labour. Geneva: World Health Organization; 2014. (http://www.who.int/reproductivehealth/publications/ maternal_perinatal_health/augmentation-labour/en/).

3. WHO recommendations on health promotion interventions for maternal and newborn health. Geneva: World Health Organization; 2015. (http:// www.who.int/maternal_child_adolescent/documents/health-promotioninterventions/en/)

4. Tuncalp Ö, Were WM, MacLennan C, Oladapo OT, Gulmezoglu AM, Bahl R, Daelmans B, Mathai M, Say L, Kristensen F, Temmerman M, Bustreo F. Quality of care for pregnant women and newborns - the WHO vision. BJOG. 2015;122:1045-9. http://onlinelibrary.wiley.com/doi/10.1111/14710528.13451/pdf

5. Kopplin E, Torres-Pereyra J, Pena V, Salinas R. Impact of psychosocial supports during childbirth: the decrease of caesarean and bonuses of the process. Pediatr Res. 2000:47:834

6. Dickinson J, Paech M, McDonald SJ, Evans SF. The impact of intrapartum analgesia on labor and delivery outcomes in nulliparous women. Aust N Z J Obstet Gynaecol. 2002;42:65-72.

7. Dickinson J, Paech M, McDonald SJ, Evans SF. Maternal satisfaction with childbirth and intrapartum analgesia in nulliparous labour. Aust N Z J Obstet Gynaecol. 2003:43:463-8

8. Gagnon AJ, Waghon K, Covell C. A randomized trial of one-to-one nurse support of women in labor. Birth. 1997;24:71-7.

9. Gagnon AJ, Waghorn K. One-to-one nurse labor support of nulliparous women stimulated with oxytocin. J Obstet Gynecol Neonatal Nurs. 1999;28:371-6.

10. Breart G, Mlika-Cabane N, Thornton J, Trakas D, Alexander S. European trials on artificial rupture of membranes and professional support during labour. J Perinat Med. 1992:20:37.

11. Hodnett ED, Osborn RW. Effects of continuous intrapartum professional support on childbirth outcomes. Res Nurs Health. 1989;12:289-97.

12. Klaus MH, Kennell JH, Robertson SS, Sosa R. Effects of social support during parturition on maternal and infant morbidity. Br Med J. 1986; 293:585-7.

13. Hemminki E, Virta A, Koponen P, Malin M, Kojo-Austin H, Tuimala R. A trial on continuous human support during labor: feasibility, interventions and mothers' satisfaction. J Psychosom Obstet Gynaecol. 1990;11:239-50.

14. McGrath SK, Kennell JH. A randomized controlled trial of continuous labor support for middle-class couples: effect on cesarean delivery rates. Birth. 2008;35(2):92-7.

15. Hodnett ED, Lowe NK, Hannah ME, Willan AR, Stevens B, Weston JA, et al. Effectiveness of nurses as providers of birth labor support in north American hospitals: a randomized controlled trial. JAMA. 2002;288:1373-81.

16. Madi BC, Sandall J, Bennett R, MacLeod C. Effects of female relative support in labor: a randomized controlled trial. Birth. 1999;26:4-8.

17. Hofmeyr JG, Nikodem VC, Wolman WL, Chalmers BE, Kramer T. Companionship to modify the clinical birth environment: effects on progress and perceptions of labour, and breastfeeding. Br J Obstet Gynaecol. 1991;98:756-64.

18. Kennell J, Klaus M, McGrath S, Robertson S, Hinkley C. Continuous emotional support during labor in a US hospital. A randomized controlled trial. JAMA. 1991:265:2197-201.

19. Morhason-Bello IO, Adedokun BO, Ojengbede OA, Olayemi O, Oladokun A, Fabamwo AO. Assessment of the effect of psychosocial support during childbirth in Ibadan, south-west Nigeria: a randomised controlled trial. Aust N Z J Obstet Gynaecol. 2009:49:145-50.

20. Cogan R, Spinnato JA. Social support during premature labor: effects on labor and the newborn. J Psychosom Obstet Gynaecol. 1988;8:209-16.

21. Bruggemann OM, Parpinelli MA, Osis MJ, Cecatti JG, Neto ASC. Support to woman by a companion of her choice during childbirth: a randomized controlled trial. Reprod Health. 2007:4:5.

22. Campbell DA, Lake MF, Falk M, Backstrand JR. A randomized control trial of continuous support in labor by a lay doula. J Obstet Gynecol Neonatal Nurs. 2006;35:456-64

23. Campbell D, Scott $K$, Klaus M, Falk M. Female relatives or friends trained as labor doulas: outcomes at 6 to 8 weeks postpartum. Birth. 2007;34:220-7. 
24. Langer A, Campero L, Garcia C, Reynoso S. Effects of psychosocial support during labor and childbirth on breastfeeding, medical intervention, and mothers' wellbeing in a Mexican public hospital;: a randomized clinical trial. Br J Obstet Gynaecol. 1998;105:1056-63.

25. Yuenyong $\mathrm{S}, \mathrm{O}$ 'Brien $B$, Jirapeet $\mathrm{V}$. Effects of labor support from close female relative on labor and maternal satisfaction in a Thai setting. J Obstet Gynecol Neonatal Nurs. 2012;41:45-56.

26. Kashanian M, Javadi F, Haghighi MM. Effect of continuous support during labor on duration of labor and rate of cesarean delivery. Int J Gynecol Obstet. 2010;109:198-200.

27. Brown H, Hofmeyr GJ, Nikodem VC, Smith H, Garner P. Promoting childbirth companions in South Africa: a randomised pilot study. BMC Med. 2007:5:7.

28. Gordon NP, Walton D, McAdam E, Derman J, Gallitero G, Garrett L. Effects of providing hospital-based doulas in health maintenance organization hospitals. Obstet Gynecol. 1999:93:422-6.

29. Lindow SW, Hendricks MW, Thompson JW, van der Spuy ZM. The effect of emotional support on maternal oxytocin levels in laboring women. Eur J Obstet Gynecol Reprod Biol. 1998;79:127-9.

30. McGrath S, Kennell J, Suresh M, Moise K, Hinkley C. Doula support vs epidural analgesia: impact on cesarean rates. Pediatr Res. 1999;45:16A.

31. Manning OG. A birth intervention: the therapeutic effects of doula support versus Lamaze preparation on first-time mothers' working models of caregiving. Alternative Therapies in Medicine. 1998:4:73-81.

32. Scott KD, Klaus PH, Klaus MH. The obstetrical and postpartum benefits of continuous support during childbirth. J Women's Health Gen based Med. 1999:8:1257-64

33. Sosa R, Kennell JH, Klaus MH, Robertson S, Urrutia J. The effect of a supportive companion on perinatal problems, length of labor and motherinfant interaction. N Engl J Med. 1980;303:597-600

34. Trueba G, Contreras C, Velazo MT, Lara EG, Martinez HB. Alternative strategy to decrease cesarean section: support by doulas during labor. J Perinat Educ. 2000;9:8-13.

35. Tryon PA. Use of comfort measures as support during labor. Nurs Res. 1966; 15:109-18

36. Thomassen P, Lundwall M, Wiger E, Wollin L, Uvnas-Moberg K. Doula- a new concept in obstetrics. Lakartidningen. 2003:100:4268-71.

37. El-Nemer A, Down S, Small N. "she would help me form the hear": an ethnography of Egyptian women in labour. Soc Sci Med. 2006;62:81-92.

38. Kumbani L, Biune G, Chirwa E, Malata A, Oyvind OJ. Why some women fail to give birth at health facilities: a qualitative study of women's perceptions of perinatal care from rural southern Malawi. Reprod Health. 2013;10:9.

39. Maimbolwa MC, Sikazwe N, Yamba B. Views on involving a social support person during labor in Zambian maternities. J Midwifery Women's Health. 2001;46:226-34.

40. Mosallam M, Rizk D, Thomas L, Eximakhai M. Women's attitudes towards psychosocial support in labour in United Arab Emirates. Arch Gynecol Obstet. 2004:269:181-7.

41. Campero L, Garcia C, Diaz C, Ortiz O, Reynoso S, Langer A. "alone, I wouldn't have known what to do": a qualitative study on social support during labor and delivery in Mexico. Soc Sci Med. 1998;47:395-403.

42. Al-Mandeel HM, Almufleh AS, Al Damri AJ, Al-Bassam DA, Hajr EA Bedawaiwi NA, Alshehri SM. Saudi women's acceptance and attitudes towards companion support during labor: should we implement an antenatal awareness program? Ann Saudi Med. 2013;33:28-33.

43. Kabakian-Khasholian T, El Nemer A, Bashour H. Perceptions about labor companionship at public teaching hospitals in three Arab countries. Int J Gynecol Obstet. 2015;129:223-6.

44. Qian X, Smith H, Zhou L, Liang J, Garner P. Evidence-based obstetrics in four hospitals in China: an observational study to explore clinical practice, women's preferences and provider's views. BMC Pregnancy and Childbirth. 2001;1:1.

45. Alexander A, Mustafa A, Emil SAV, Amekah E, Engmann C, Adanu R, Moyer CA. Social support during delivery in rural central Ghana: a mixed methods study of women's preferences for and against inclusion of a lay companion in the delivery room. J Biosoc Sci. 2014;46:669-85.

46. Banda G, Kafulafula G, Nyirenda E, Taulo F, Kalilani L. Acceptability and experience of supportive companionship during childbirth in Malawi. BJOG: An International Journal of Obstetrics and Gynaecology. 2010;117:937-45.

47. The SURE Collaboration. SURE guides for preparing and using evidencebased policy briefs: identifying and addressing barriers to implementing policy options. 2011. Version 2.1. (http://epoc.cochrane.org/sites/epoc.
cochrane.org/files/public/uploads/SURE-Guides-v2.1/Collectedfiles/sure_ guides.html).

48. Davis Harte J, Sheehan A, Stewart S, Foureur M. Childbirth supporters' experiences in a built hospital birth environment: exploring inhibiting and facilitating factors in negotiating the supporter role. Health Environment Research \& Design Journal. 2016;9:135-61.

\section{Submit your next manuscript to BioMed Central and we will help you at every step:}

- We accept pre-submission inquiries

- Our selector tool helps you to find the most relevant journal

- We provide round the clock customer support

- Convenient online submission

- Thorough peer review

- Inclusion in PubMed and all major indexing services

- Maximum visibility for your research

Submit your manuscript at www.biomedcentral.com/submit
C) Biomed Central 